\title{
Computational Chemistry for Chemistry Educators
}

\author{
Shawn C. Sendlinger \\ North Carolina Central University \\ Department of Chemistry \\ 1801 Fayetteville Street, \\ Durham, NC 27707 \\ 919-530-6297 \\ ssendlin@nccu.edu
}

\author{
Clyde R. Metz \\ College of Charleston \\ Department of Chemistry and Biochemistry \\ 66 George Street, \\ Charleston, SC 29424 \\ 843-953-8097 \\ metzc@cofc.edu
}

\begin{abstract}
In this paper we describe an ongoing project where the goal is to develop competence and confidence among chemistry faculty so they are able to utilize computational chemistry as an effective teaching tool. Advances in hardware and software have made research-grade tools readily available to the academic community. Training is required so that faculty can take full advantage of this technology, begin to transform the educational landscape, and attract more students to the study of science.
\end{abstract}

\section{Categories and Subject Descriptors}

J.2 [Physical Sciences and Engineering]: Chemistry

\section{General Terms}

Algorithms, Design, Experimentation, Theory, Verification.

\section{Keywords}

Computational Chemistry Education, Workshops, Graduate, Undergraduate.

\section{INTRODUCTION}

The majority of today's students are technologically savvy and are often more comfortable using computers than the faculty who teach them. In order to harness the student's interest in technology and begin to use it as an educational tool, most faculty members require some level of instruction and training. Because chemistry research increasingly utilizes computation as an important tool, our approach to chemistry education should reflect this. The ability of computer technology to visualize and manipulate objects on an atomic scale can be a powerful tool to increase both student interest in chemistry as well as their level of understanding. Computational Chemistry for Chemistry Educators (CCCE) is a project that seeks to provide faculty the necessary knowledge, experience, and technology access so that they can begin to design and incorporate computational approaches in the courses they teach. We also work from the viewpoint that technology should enhance the educational experience and allow teaching of concepts in new and more effective ways. In other words, just because something can be done computationally does not necessarily mean that it should be.

This project takes a broad view of what computational chemistry entails. In addition to molecular modeling, the general use of computer technology to demonstrate and help students learn chemical principles is included. This includes visualization, animation, data manipulation and graphing, system dynamics software, computer algebra systems, etc.

\section{DESCRIPTION}

Initial planning for a one week workshop that would provide hands-on instruction in the use of molecular modeling software began in 2002 with support from the Shodor Education Foundation through the National Computational Science Institute (NCSI) program, a project supported in large part by the National Science Foundation (NSF). ${ }^{1}$ Subsequent support has been provided by the Supercomputing (SC) Education Program ${ }^{2}$ and most recently by the NSF-supported Center for Workshops in the Chemical Sciences (CWCS) project. $^{3}$

The CCCE workshop was the first in a series of NCSI disciplinespecific computational science workshops, and provided a template for those that followed. ${ }^{4}$ The topics chosen for the molecular modeling workshop were:

personal or classroom use is granted without fee provided that copies are not made or distributed for profit or commercial advantage and that copies bear this notice and the full citation on the first page. To copy otherwise, or republish, to post on servers or to redistribute to lists, requires prior specific permission and/or a fee. Copyright CJOCSE, a supported publication of the Shodor Education Foundation Inc.
1. Introduction to Computational Chemistry

2. Basis Sets

3. Choice of Theoretical Method 
4. Single Point Energies and Geometry Optimization

5. Electron Densities, Electrostatic Potentials, and Reactivity Predictions

6. Modeling in Solution

7. Computing Spectroscopic and Thermochemical Properties

8. Quantitative Structure Activity/Property Relationships

9. Transition States

10. Computational Study of System Dynamics (Chemical Kinetics)

11. Biochemical Applications

Each session consists of a lecture followed by hands-on computer exercises that illustrate the lecture concepts. ${ }^{5}$ These sessions provide the participants with sufficient breadth and depth so that by the end of the week they are able to choose an appropriate theoretical model chemistry and basis set, and also know how to set up, perform, and interpret calculations. Participants are not expected to become expert quantum chemists! This is not the goal of the workshop. We seek to provide faculty with a level of molecular modeling knowledge so that they can competently use the tool and have confidence in the results of their calculations. In particular, we hope that the participants are able to answer the following questions after the workshop:

a) What do I want to know about a molecular or ionic system?

b) How accurately do I need to know it?

c) How long am I willing to wait for the answer to be computed?

d) What software/hardware can I use to accomplish the task?

The answers to the above questions then dictate the possible computational approaches that should be used. The level of knowledge the participants should gain is similar to that of many modern spectroscopic techniques that are regularly employed by chemists. One does not need to be an expert in the mathematics and quantum mechanics involved in nuclear magnetic resonance (NMR) spectroscopy to be able to correctly interpret the results of NMR experiments. However, we believe that the user should also be beyond the "black box" level of understanding, and have a firm grasp of what a given technique can or cannot do. We believe that the topics are addressed in sufficient detail for the participants to attain the desired level of expertise.

In addition to the molecular modeling-focused topics, a session on chemical kinetics is included. Spreadsheet-based activities are incorporated, but the main hands-on portion of the exercise is based on systems dynamics software ${ }^{6}$ with which many faculty members are unfamiliar. We also mention computer algebra systems (Mathcad, Matlab, Mathematica, etc.) and include example code so that those with access to one of these programs could begin to build their own chemical kinetics model.

Other non-molecular modeling sessions focus on free or low-cost software, browser plug-ins such as molecular viewers, and online repositories of information that have proven useful for educational purposes. These sessions are either hands-on or demonstrations that explore the use of each resource. Examples include:

Free molecular drawing programs

ACD ChemSketch/3D: http://www.acdlabs.com/download/

BioRad KnowItAll: http://www.knowitall.com/academic/

Web-Based Resource Collections

Computational Science Education Reference Desk (CSERD): http://www.shodor.org/refdesk/
Interactivate: http://www.shodor.org/interactivate/

Virtual Lab Simulator:

http://www.chemcollective.org/vlab/vlab.php

Netlogo: http://ccl.northwestern.edu/netlogo/

ReciprocalNet: http://www.reciprocalnet.org/

Protein Data Bank: http://www.rcsb.org/pdb/home/home.do

Journal of Chemical Education: http://jchemed.chem.wisc.edu/

Proteopedia: http://www.proteopedia.org

Viewers for molecular structure

CHIME:

http://www.symyx.com/support/developer/chime/index.jsp

VRML: http://www.parallelgraphics.com/products/cortona3d/

Jmol: http://jmol.sourceforge.net/

Mercury: http://www.ccdc.cam.ac.uk/free_services/mercury/

Freely available Graphical User Interfaces, computational engines, and Viewers for molecular modeling

RUNpcg: http://www.chemsoft.ch/qc/RUNpcg.htm

Firefly: http://classic.chem.msu.su/gran/gamess/

Molekel: http://molekel.cscs.ch/wiki/pmwiki.php

Workshop participants apply their new knowledge as they begin to develop a case study that they will use in a course that they regularly teach. Time to work on the case study is included in the daily workshop schedule. The workshop culminates with presentations of these case studies on the final day. Some of the developed case studies are appropriate for use during several minutes of lecture time, while others are designed to occupy several hours during a laboratory period. Many of these projects focus on the use of computation to teach old topics in a new (and hopefully, more effective) manner. Others introduce students to molecular modeling and instruct students on how to build molecules, perform calculations, and interpret their own results. Some are designed as homework assignments that students complete outside of class on their own time. One very effective educational approach is to use a series of computations to provide results that the students then must interpret, identify trends, and make sense of the trend using the knowledge of chemistry that they have gained to that point. This discovery-mode of learning can help students to understand and retain the information more effectively. ${ }^{\text {? }}$

Other week-long workshops have been developed and taught at both the Introductory and Advanced levels. Introductory workshops place less emphasis on molecular modeling and focus more on the other topics mentioned. The Advanced workshops cover molecular modeling subjects of interest to the attendees in more detail and provide additional time for collaboration among the attendees to develop classroom materials.

A number of shorter workshops have also been provided. These range from half-day sessions at local and regional ACS meetings ${ }^{8}$ or Supercomputing Education Program events ${ }^{9}$ to full- or multiple-day sessions arranged with individual institutions.

\section{RESULTS}

\subsection{Workshops}

Since 2002, the week-long molecular modeling workshop has been taught eight times with $\sim 165$ attendees. Advanced and Introductory workshops have been taught twice each with a total of $\sim 60$ participants. Thirteen shorter workshops that cover the 
basics of molecular modeling and other topics have also been held with over 200 participants attending. Computational chemistry has also been included as a major part of an ongoing high school teacher development project in Illinois with over 120 participants. ${ }^{10}$ Over the past seven years, the CCCE project has thus reached well over 500 college faculty and high school teachers. We hope to continue our work and further increase these numbers.

Workshop participants and instructors are active in presenting their work at various professional meetings ${ }^{11}$ and in appropriate journals. ${ }^{12}$ This is an expected outcome of workshop attendance and we hope that the current journal will become a popular venue for sharing successful activities.

Teaching these workshops has led the authors to initiate new courses at their own institutions. The College of Charleston has both Introduction to Modeling in Chemistry and Advanced Physical Chemistry: Molecular Modeling courses. North Carolina Central University has an interdisciplinary, team-taught Introduction to Computational Science and Informatics course as well as a graduate-level Computational Chemistry course. Computational content has also been added in other courses where appropriate.

\subsection{Workshop Assessment}

Each workshop was evaluated using three different types of anonymous on-line evaluation forms. A pre-workshop survey identified the level of current knowledge and available resources before the workshop began. Daily evaluations helped the instructors determine participant progress and provided sessionspecific feedback. A post-workshop survey evaluated overall advancement in knowledge and gave an indication of the level of competence and confidence in using computational tools that the participants had gained.

The post-workshop survey consisted of a series of questions to which the participant could respond with simple "yes or no" answers, rate the response over a numerical scale, and generate free-style short responses. The following example questions and responses were chosen from 25 completed surveys by college faculty for the 2008 and 2009 molecular modeling workshops. To simplify the presentation of results, numerical scores were assigned to the various responses as indicated below and the average score is given with the standard deviation noted in parentheses.

Scoring for questions 1-3: [strongly disagree $=0$, somewhat disagree $=1$, somewhat agree $=2$, strongly agree $=3$ ]

1. Did the workshop advance the participant's knowledge of computational science? $2.56(0.90)$

2. Did the participant learn to use computational science in teaching? $\underline{2.52}(0.85)$

3. Did the participant learn to use models applicable to chemistry? $\underline{2.36}(0.84)$

4. Did the participant learn to use models to demonstrate a concept in a course? Affirmative for $\underline{96 \%}$ of the responses

5. Will the participant work with individual instructors at the home institution to assist them to learn more about computational science education activities? Affirmative for $\underline{88 \%}$ of the responses
6. The self-rated confidence level on using computational science to enhance the learning experience of the students [not at all $=1$, very confident $=10]$ of the participants was $\underline{8.24}(1.63)$

The above results combined with verbal and e-mail comments made by participants during and following the workshops indicate substantial gains in both knowledge and confidence for using computational approaches in the classroom.

\subsection{Related Accomplishments}

The authors have also become involved in several related projects. UNChem is an online chemistry fundamentals review course ${ }^{13}$ designed for students who will begin freshman chemistry. The site is being reviewed and updated. Many of the resources used in the CCCE workshops will be incorporated into the materials. The Computational Science Education Reference Desk (CSERD), ${ }^{14}$ a Pathways project of the National Science Digital Library ${ }^{15}$ and funded by the NSF, aims to help students learn about computational science and to help teachers incorporate it into the classroom. The authors and workshop attendees have edited and reviewed much of the chemistry content of CSERD.

A very useful workshop product has been the CCCE web site available at: http://www.computationalscience.org/ccce/. The site currently houses the molecular modeling workshop lecture and laboratory materials, a reference book list, a link to a glossary of computational chemistry terms, and links to other chemistry resources. Site visitors most often utilize the Labs link (shown on the next page) which has a matrix of ten molecular modeling topics that have been translated into exercises that can be performed with seven different popular molecular modeling programs. The exercises contain scaffolded instructions which, when followed in sequence, teach the user how to effectively use the software package and to also gain experience with different computational methods. Also included are instructions in the use of spreadsheets, computer algebra software, and systems dynamics programs for the modeling of chemical kinetics.

In the fall of 2009, Computational Chemistry for Chemistry Educators was awarded the Undergraduate Computational Engineering and Science (UCES) Award. This award program was created to promote and enhance undergraduate education in computational engineering and science and encourages further development of innovative educational resources and programs, recognizes the achievements of CES educators, and disseminates educational material and ideas to the broad scientific and engineering undergraduate community. The UCES Awards Program is funded by the Department of Energy and administered by the Krell Institute. ${ }^{16}$

\subsection{Technology}

The first several years of the CCCE program used PC-based molecular modeling software, such as CAChe, ${ }^{17}$ Spartan, ${ }^{18}$ Hyperchem, ${ }^{19}$ or PC Model $^{20}$ for all hands-on exercises. This approach necessitated loading (and troubleshooting) many programs and license files on multiple machines. In order for participants to have software to work with when they returned to their home institution, a mini-grant program was used where participants would apply for funds to cover the cost of a software package, a computer RAM or HD upgrade, plus a few books. In 2006 we began to use $\mathrm{WebMO}^{21}$ which is a server-based GUI that 
allows drawing molecules, setting up and submitting jobs, and visualizing the results. The WebMO software interfaces to computational engines such as MOPAC, ${ }^{22}$ Gaussian, ${ }^{23}$ Tinker, ${ }^{24}$ and NWChem. ${ }^{25}$ The switch to a server-based system greatly reduced the individual computer set-up time and also allows participants to have continued access to their files following the workshop. This thin client model also makes it easy to provide the students of our workshop participants with access to the software as well. It is, after all, the students and their learning outcomes that we want to promote.

\section{FUTURE WORK}

As individual software packages undergo periodic updates, the exercise instructions provided on the CCCE site must be changed. A new and improved version of the site is being developed and will include new instructions where needed, along with desktopcapture video instructions on using different software programs. We also hope to replace the current, static lecture materials with information that is more interactive and includes some video and voiceover.

For the past eight years, a main thrust of CCCE has been focused on electronic structure calculations for single molecules. This focus is largely a result of the hardware and software that has been readily available. Our move to the use of server-based WebMO removes some of the hardware constraints and allows us to begin planning for the inclusion of more reaction dynamics and molecular dynamics calculations and exercises. The workshops currently use the Odyssey software package ${ }^{26}$ for molecular dynamics exercises. While this package is quite useful for educational purposes, it is a commercial product and many workshop attendees cannot afford it. Researchers use molecular dynamics programs such as freely available GROMACS ${ }^{27}$ and NAMD. ${ }^{28}$ To extend the CCCE model in this direction will require a GUI, several of which are available, ${ }^{29}$ and hardware on which to perform the simulations. Work to provide these resources in ongoing. As ever-larger computing resources come online, such as the Blue Waters petascale machine, ${ }^{30}$ CCCE hopes to assist faculty and teachers in learning how to use these systems in a scientifically sound and educationally productive manner. It is incumbent upon the educators of today to motivate and train our students to become the scientists of tomorrow. Computational modeling can help interest more students in science and also provide the skills our graduates will need to harness the power of future computer technology.

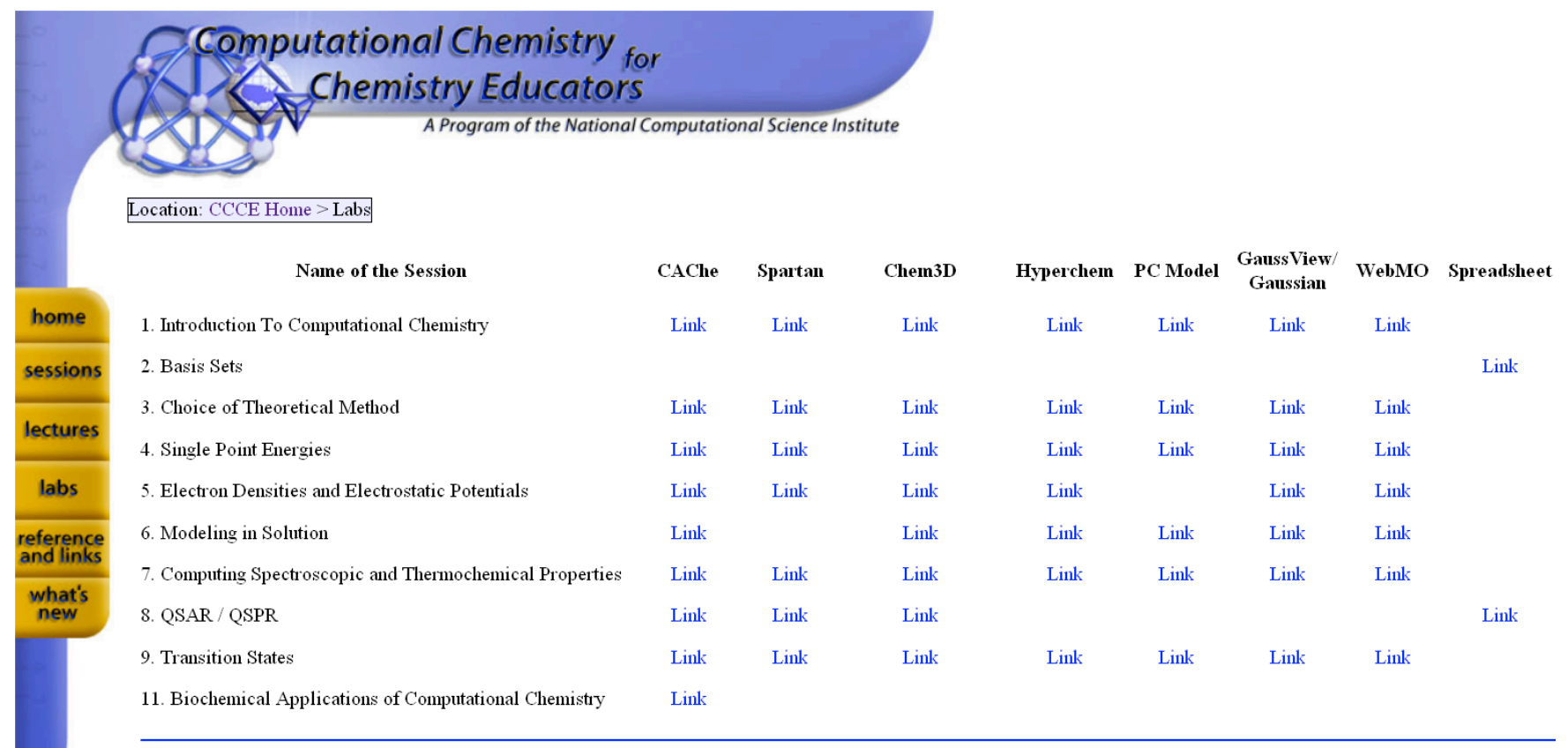

\section{ACKNOWLEDGEMENTS}

The authors wish to thank Dr. Robert M. Panoff, Executive Director and President of the Shodor Education Foundation for his years of support and encouragement, Mr. Bob Gotwals of the North Carolina School for Science and Mathematics for his assistance in the initial workshop planning stages, Dr. Elizabeth Bell-Loncella of the University of Pittsburgh at Johnstown for recently joining the instructional team, and Dr. Brad Stone of San Jose State University for having hosted four CCCE workshops.
Dr. Jay Mashl of the National Center for Supercomputing Applications at the University of Illinois at Urbana-Champaign and Charlie Peck of Earlham College have provided invaluable software and hardware support. We would also like to thank the other workshop hosts as well as the funding agencies whose support made this work possible: NSF DUE 0127488, SC Education Program, and Dr. Jerry Smith with the Center for Workshops in the Chemical Sciences (NSF CCLI-ND). 


\section{REFERENCES}

[1] National Computational Science Institute: http://computationalscience.org/.

[2] SC Education Program: http://sc10.sc-education.org/.

[3] Center for Workshops in the Chemical Sciences: http://chemistry.gsu.edu/CWCS/.

[4] Other workshops are available in Biology, Computational Thinking, Parallel and Cluster Computing, and Physics. See: http://www.computationalscience.org/workshops/.

[5] For additional details, see the CCCE web site at: http://www.computationalscience.org/ccce/.

[6] http://www.vensim.com/sdmail/sdsoft.html.

[7] Altintas, I.; Berkley, C.; Jaeger, E.; Jones, M.; Ludascher, B.; Mock, S., "Kepler: An Extensible System for Design and Execution of Scientific Workflows," Proceedings of the 16th Int'l Conf. Scientific and Statistical Database Management, IEEE Press, 2004, pp. 423-424.

[8] For college and university faculty: http://acs.confex.com/acs/56serm/techprogram/S261.HTM. For high school teachers: http://acs.confex.com/acs/56serm/techprogram/S953.HTM

[9] http://moodle.sc-education.org/.

[10] Institute for Chemistry Literacy through Computational Science (ICLCS): http://iclcs.uiuc.edu/.

[11] Symposia:

http://acs.confex.com/acs/56serm/techprogram/S281.HTM. Faculty workshops:

http://acs.confex.com/acs/56serm/techprogram/S261.HTM. Teacher workshops: http://acs.confex.com/acs/56serm/techprogram/S953.HTM.

[12] (a) Sendlinger, S.C. and Metz, C.R., "CSERD: Another Important NSDL Pathway for Computational Chemistry Education", J. Chem. Ed., 2009, 86, p. 126. (b) Sendlinger, S.C.; DeCoste, D.J.; Dunning, T.H.; Dummitt, D.A.;
Jakobsson, E.; Mattson, D.R.; Wiziecki, E.N., "Transforming Chemistry Education through Computational Science", Computing in Science and Engineering, 2008, 10(5), pp. 3439.

[13] http://www.shodor.org/unchem/.

[14] http://www.shodor.org/refdesk/.

[15] http://nsdl.org/.

[16] http://www.krellinst.org/csgf/awards-contests/uces-awardprogram

[17]

http://solutions.us.fujitsu.com/www/content/news/newsdetail .php?nf $=07230560$. nitf

[18] http://www.wavefun.com/products/spartan.html.

[19] http://www.hyper.com/.

[20] http://www.serenasoft.com/.

[21] http://www.webmo.net/.

[22] http://openmopac.net/home.html.

[23] http://www.gaussian.com/.

[24] http://dasher.wustl.edu/tinker/.

[25] http://www.nwchem-sw.org/index.php/Main Page.

[26] http://www.wavefun.com/products/odyssey/odyssey.html.

[27] http://www.gromacs.org/.

]28] http://www.ks.uiuc.edu/Research/namd/.

[29] (a) Roopra, S.; Knapp, B; Omasits, U.; Schreiner, W., "jSimMacs for GROMACS: A Java Application for Advanced Molecular Dynamics Simulations with Remote Access Capability", J. Chem. Inf. Model., 2009, 49(10), pp. 2412-2417. (b) Sellis, D.; Vlachakis, D.; Vlassi, M., "Gromita: A Fully Integrated Graphical User Interface to Gromacs 4", Bioinformatics and Biology Insights, 2009, 3, pp. 99-102. (c) http://kdeapps.org/content $/$ show.php $/ \% 20$ Gromacs + GUI + ?content $=47$ 665 .

[30] http://www.ncsa.illinois.edu/BlueWaters/. 